\title{
La rhétorique de la congolité dans la publicité en RD \\ Congo. Enjeux d'un discours de populisme nationaliste en Afrique francophone
}

\author{
Jean-Claude MAPENDANO BYAMUNGU1
}

\begin{abstract}
The field of advertising is a melting pot of ideologies, that is, of both cultural and political identities. Today, these are built in and through social discourses specific to the contemporary context. In DR Congo, the instrumentation of the Congolese paradigm with a hint of sovereignty has increasingly become a constant in the advertising discourse. It is a media dynamic of expression of a certain autarky against Western and Sino-American economic imperialism, through the promotion of the local industry. It is deployed through the rhetoric of "Congolity " which is understood in terms of a catalyst for the issues of a discourse of nationalist populism in a country of French-speaking sub-Saharan Africa.
\end{abstract}

Keywords: argumentation; discourse analysis; Congolity; nationalist populism; advertising

DOI: 10.24818/DLG/2021/38/09

\section{Introduction}

L

es discours politiques d'Afrique sont aujourd'hui marqués d'un élan souverainiste en guerre ouverte contre l'impérialisme à la fois culturel, économique et politique aussi bien européen que sinoaméricain. En effet, les thèmes de «souveraineté » et $\mathrm{d}^{\prime}$ " identités » nationales sont devenus des constantes des divers discours sociaux enclins au populisme qui gagne de plus en plus du terrain un peu partout dans le monde. Ainsi le discours publicitaire spécifique au contexte sociopolitique et économique de la RD Congo se déploie-t-il en une rhétorique de la « congolité ». Celle-ci consiste en l'instrumentation du paradigme congolais comme stratégie de manipulation de la clientèle. Dès lors, elle constitue une « institution symbolique » de la praxis markétique dans la sémiosphère du pays.

\footnotetext{
${ }^{1}$ Jean-Claude Mapendano Byamungu, ISP de Kichanga/RD Congo, jcmapendanos26@gmail.com
} 
En d'autres termes, le concept de "congolité » en publicité, forgé sur le modèle, entre autres, de ceux d'«ivoirité » (Côte $\mathrm{d}^{\prime}$ Ivoire), de "francité » (France), d' « italianité » (Italie) ou encore de "portugalité » (Portugal) désigne non seulement le phénomène d'inscription du paradigme congolais au sens culturel du terme, mais aussi celui d'ambition politique d'exaltation d'un sentiment fort de défense d'un patrimoine commun pour la gloire de la nation. Il relève d'un activisme qui a émergé depuis le lancement en 2013 de la campagne "Consommons congolais" comme désormais la politique régulatrice du marché économique. D'ailleurs, un Fonds de Promotion de l'Industrie (FPI) a été mis en place pour la promotion de l'industrie locale, à travers notamment le financement des projets du secteur privé. Le discours qui le porte est un discours d'empreinte et d'emprise dont l'enjeu repose sur la coconstruction d'un ethos collectif de "producteur/consommateur" congolais nationaliste.

Les travaux sur les paradigmes culturels et identitaires remontent, entre autres, à Charaudeau, P. (2001), Dion, D. et al. (2010), Sintas, S. (2013), Yücel, H. (2013), Eloundou Eloundou, V. (2013), Harir, D et Bakrim, N. (2013). Dans l'ensemble, il s'agit des travaux qui s'inscrivent tous dans le champ théorique et épistémologique global de la Sémantique des Possibles Argumentatifs (SPA), développé par Galatanu, O. (2003/2009), à l'exception des travaux de Charaudeau (2001) et de Vion (2010). La SPA est une perspective formulée dans une démarche de l'Analyse Linguistique du Discours (ALD), conçue comme «le repérage et l'étude des mécanismes sémantico-discursifs pragmatico-discursifs de production et d'interprétation du sens » (Galatanu, O., 1999). Elle s'inscrit dans une filiation de la sémantique argumentative (Anscombre et Ducrot, 1983 et 1995 ; Carel, 1995) ainsi que des recherches sur les stéréotypes linguistiques (Putnam, 1975 et 1990 ; Fradin, 1994 ; Kleiberg, 1999).

Nous envisageons la "congolité », une virtualité épistémologique, non seulement comme un construit discursif mais aussi et surtout comme un paradigme énonçable, porteur d'un pouvoir fort persuasif dans le processus markétique. Ainsi la présente étude consiste-t-elle à décrire les mécanismes sociodiscursifs et sémantiques d'instrumentation de cette «congolité », par lesquels le discours publicitaire entend modeler en l'orientant la décision du destinataire-client/consommateur pour l'achat des produits de marque «made in DR Congo » que l'on lui propose dans le jeu interlocutif de la communication marchande. De ce point de vue, notre 
problématique située à l'interface de la rhétorique identitaire et de la souveraineté politique (Mohammedi, A. 2017) sous-tend les interrogations ci-après : Quelles sont les formes sémiotiques d'énonciation de la congolité dans le discours publicitaire? En quoi les mécanismes sociodiscursifs et sémantiques mis en jeu sous-tendent la co-construction d'un ethos collectif de « producteur-consommateur » nationaliste congolais.

$\mathrm{Au}$ regard de ces interrogations, notre ancrage théorique est sans doute celui d'une approche interdisciplinaire, en l'occurrence l'« analyse du discours à la française » (Maingueneau, 1995), en tant qu'elle articule les divers genres de discours à leurs contextes de production. Celle-ci a été définie par Maingueneau (2009 : 18-19) en ce termes :

"Pour notre part (Maingueneau, 1995), nous jugeons préférable de spécifier l'analyse du discours comme la discipline qui, au lieu de procéder à une analyse linguistique du texte en lui-même ou à une analyse sociologique ou psychologique de son "contexte", vise à rapporter les textes, à travers leurs dispositifs d'énonciation, aux lieux sociaux qui les rendent possibles et qu'ils rendent possibles. En conséquence, l'analyse du discours accorde un rôle clé aux genres de discours, qui ne sont pas considérés comme des types de textes, dans une perspective taxinomique, mais comme des dispositifs de communication, de nature à la fois sociale et linguistique. »

C'est un appareil conceptuel et méthodologique susceptible de cerner les divers codes et réseaux de significations de la «congolité » envisagée comme ancrage de satellisation du discours. Par ailleurs, il s'agira en quelque sorte d'une investigation sémiostylistique des stratégies discursives d'un paramètre clé du discours politique congolais. Nous y interrogerons aussi bien les combinaisons des formes et leurs valeurs, le dispositif sémiotique actualisé que le système d'inférences qui ressortissent de la représentation opérée dans le processus de noyautage de la clientèle. Le corpus sous examen est constitué d'énoncés verbaux (slogans publicitaires) et d'iconotextes récoltés à partir des divers supports médiatiques tels que la radio, la télévision et l'affiche. Le corps du travail se décline en deux volets principaux à savoir les formes sémiotiques de la congolité ainsi que l'analyse de celle-ci en tant que rhétorique de populisme nationaliste au sein de la «pubosphère » de la RD Congo. 


\section{Le discours publicitaire et les mécanismes langagiers d'énonciation de la congolité}

Après avoir étayé le macro-contexte sociopolitique dans lequel émerge cette publicité aux élans nationalistes autour de la "congolité », il importe de décrire, dans un premier temps, tous les mécanismes sociodiscursifs et langagiers au prisme desquels s'énonce ce paradigme. En effet, le congolité comme «levier d'argumentation marchande » se déploie à travers deux principales formes sémiotiques à savoir les signes iconiques et linguistiques (verbaux) avec lesquels joue le publiciste pour en faire des objets c'est-à-dire des outils de communication.

\subsection{L'expression symbolique de la congolité}

La première forme sémiotique par laquelle s'énonce la «congolité » dans les métadonnées du corpus que nous analysons, ce sont les iconotextes c'est-à-dire des signes iconiques ou des images. Ces formes encodent en leur sein, des contenus connotatifs du paradigme congolais. C'est dans ce sens que nous les interprétons comme des «tropes iconiques » portées par des indices chromatiques et autres symboles qui rappellent les couleurs et les emblèmes du drapeau national congolais. Elles relèvent des différentes affiches et photos capturées pour des soucis d'exemplification. Ces types d'éléments ne sont guère arbitraires ni innocents car ils expriment une idée bien élaborée au préalable et précise. En cela, ils participent du jeu d'orientation argumentative du discours. Ces supports sémiotiques nous intéressent à bien des égards car selon Galatanu, O. (2007) :

"[...] le cadre d'une affiche publicitaire en général, est à la fois un champ de vision, un champ d'interprétation et de signification. Il rassemble dans un espace délimité, clos, un ensemble de signes. Il délimite une pensée, une visée bien précise. Il est "une matrice par représentation ».

Par ailleurs, précise encore Maingueneau (2014: 6), « un texte publicitaire, en particulier, est fondamentalement un "iconotexte ", image et parole, le verbal même faisant image ». Dans la pratique, l'exploration du corpus révèle une certaine tendance, pour les concepteurs des enseignes murales, d'arborer l'enseigne de leur établissement commercial de la trilogie chromatique bleu-jaune-rouge. Ce processus de sémiotisation de la « congolité » se retrouve dans des exemples d'enseignes telles que celle du mini-bar/buvette «Le petit bazar », celle de «La maison Mbiza», une usine de 
fabrication des matelas, ou encore l'affiche publicitaire «To lova $R D C$, to pona Primus» [Nous avons aimé la RDC, nous avons choisi Primus"], produite par la société Bralima pour sa bière Primus et que l'on retrouve peinte sur le mur d'un bar à Goma. Il s'agit des enseignes et affiches ci-dessous :

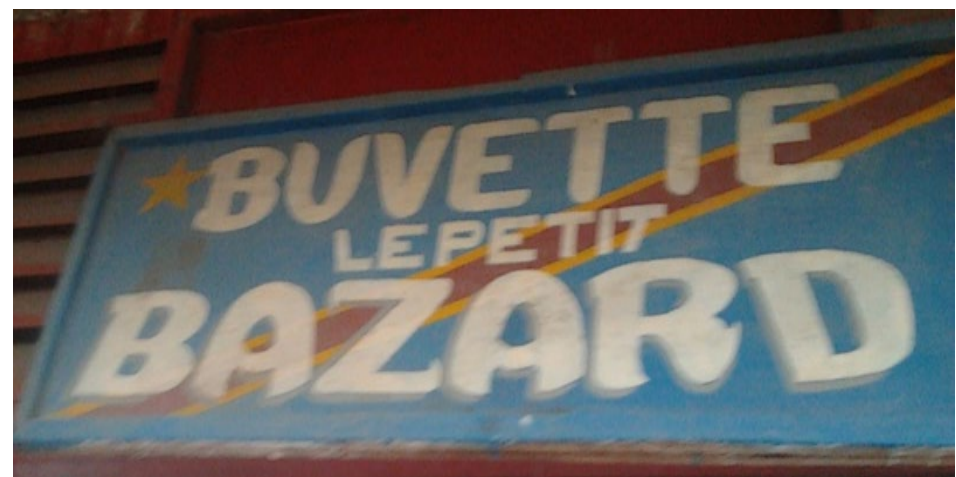

Figure 1. Enseigne murale du barre Le petit Bazard

Dans le même registre, citons cette autre enseigne murale retrouvée sur le mur d'une usine de matelas, comme établissement commercial destiné à s'inscrire dans la dynamique du Fonds de Promotion de l'Industrie (FPI) :

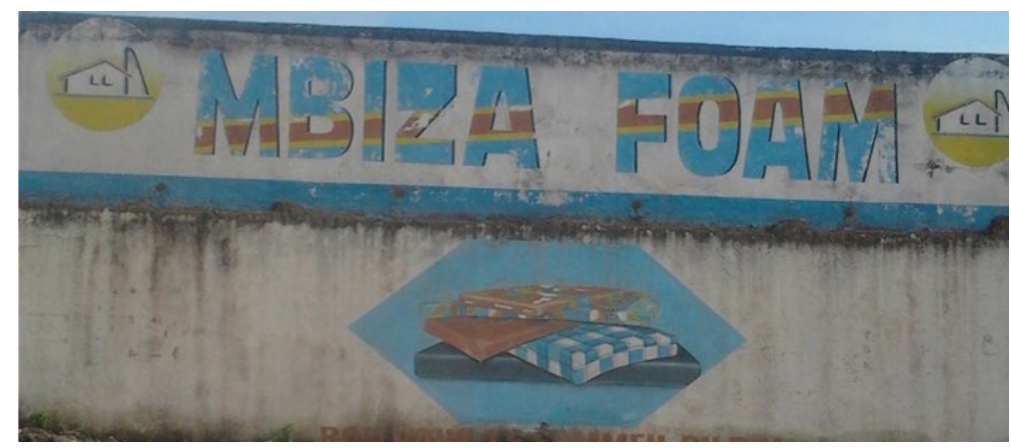

Figure 2. Enseigne murale « Mbiza Foam » de l'usine de matelas

Les mêmes signes symboliques c'est-à-dire les couleurs empruntées aux emblèmes (drapeau, armoiries) du drapeau national se retrouvent également sur l'iconotexte ci-dessous portant sur la publicité de la bière Primus. 


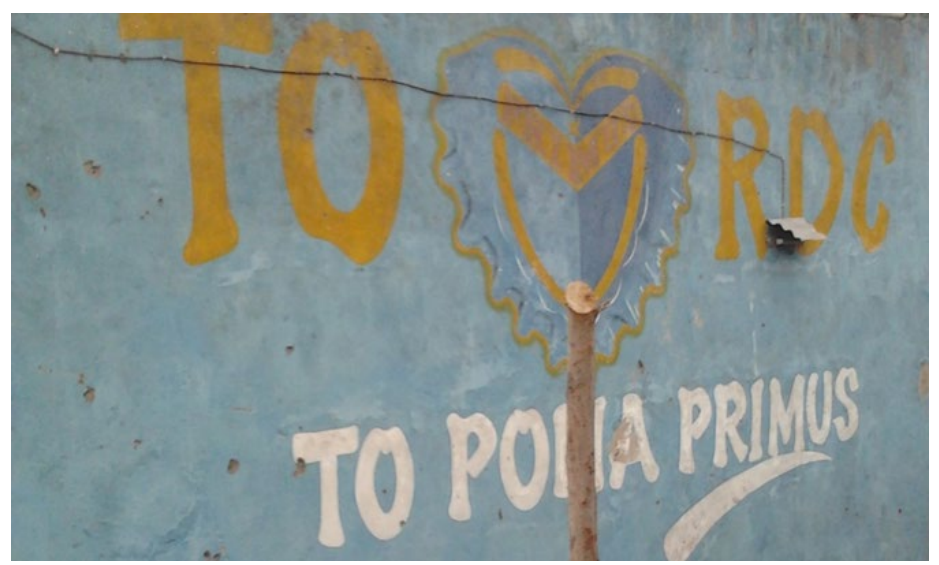

Figure 3. Affiche d'une publicité de la Bralima sur un mur

Au-delà des signifiants chromatiques, l'expression de la congolité y est également renforcée par l'énoncé verbal «To lova $R D C$, to pona Primus ». $\mathrm{Au}$ sein de cet énoncé publicitaire, le mot «lova» est sémiotiquement représenté par le symbole «Love» du langage amoureux. C'est un slogan qui pose alors l'acte de choisir la bière Primus comme un acte politique de digne citoyen, patriote et nationaliste ainsi qu'une preuve d'amour pour la nation. Pris en charge par un «Nous» collectif, l'énoncé suppose l'engagement de l'instance énonciative dans le contenu propositionnel de celui-ci et le pose ainsi comme un «lieu commun » c'est-à-dire un savoir partagé par l'opinion commune et dans la logique duquel le destinataire (client) doit s'inscrire au risque d'enfreindre une norme socialement instituée au sein de la doxa.

En même temps, le logo de la nouvelle bière Primus tout comme toutes les affiches publicitaires de la Bralima comportent ces couleurs du drapeau national congolais. Il en va des deux affiches ci-après, publiées sur la page Facebook de la Bralima. 


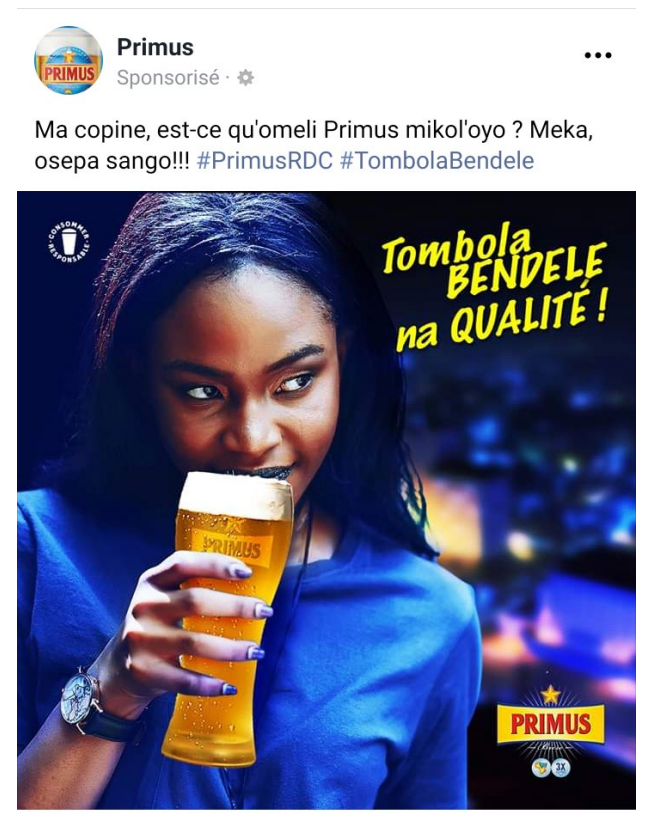

Figure 4. Affiche Primus/Bralima

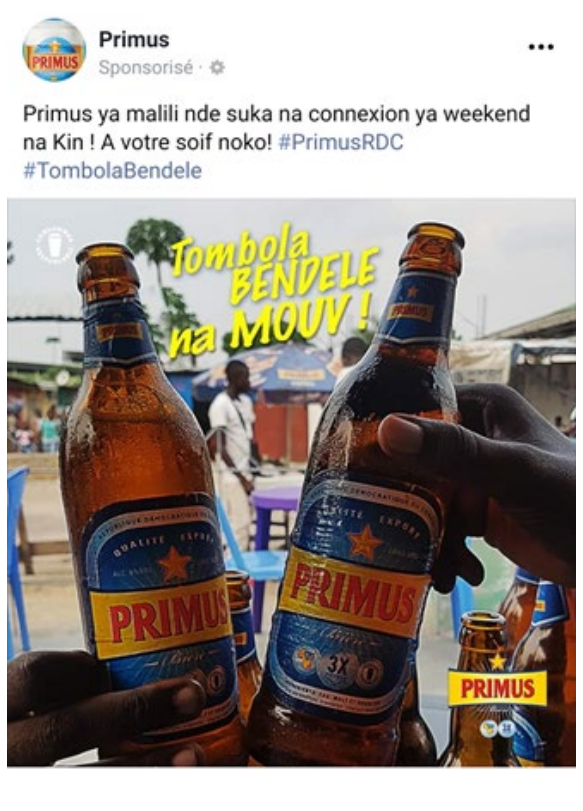

Figure 5. Affiche Primus/Bralima

Dans la Figure 4, la couleur bleue du drapeau national est représentée aussi bien dans le fond de l'image qu'à travers le T-Shirt que porte la jeune fille en train de déguster la fraicheur d'un verre de bière Primus. La couleur jaune est quant à elle manifeste à travers l'énoncé verbal «Tombola Bendele na Qualité !", la petite bande avec étoile, située en bas et à droite, dans laquelle est inscrite la marque de bière "Primus » à travers la couleur de la bière dans le verre. Enfin, la couleur rouge est remarquable à travers les lettres $\mathrm{du}$ «marconyme» (nom de la marque) «Primus» inscrit dans la bande jaune. Dans la Figure 5 mettant plus particulièrement en scène la bouteille "Primus ", par contre les signes qui représentent les mêmes couleurs sont assez visibles sur l'autocollant servant de sceau de la société Bralima. L'exaltation nationaliste qui soustend la rhétorique de la congolité est ici renforcée par le message verbal en lingala l'une des quatre langues nationales, «Tombola Bendele!», signifiant « Hissez le drapeau! ", le porte-flambeau d'une nation ».

Cependant, l'énoncé en couleur jaune a changé d'un mot pour devenir «Tombola Bendele na Mouv! ». D'ailleurs, la plupart du matériel de la Bralima (chaises plastiques, tables, tentes, chapeaux, véhicules, motos, bâtiments servant de points de vente, communément appelés dépôts relais) 
sont désormais peints en ces couleurs par lesquelles la société présente sa bière comme un produit " made in Congo », une valeur ajoutée, susceptible de décider le client à la consommer afin de bien jouir de sa congolité. Point n'est besoin de rappeler qu'il existe une certaine "idée reçue " (Amossy, 200) valorisante, selon laquelle la nationalité congolaise (RD Congo) est très convoitée par des citoyens de beaucoup d'autres pays voisins, notamment les Rwandais. Dès lors la posséder devient un signe de fierté au point qu'il faudra aussi la conserver car sa perte, sous quelque forme que ce soit, est toujours perçue comme un signe de déchéance de citoyenneté. Cette réalité constitue l'une des explications du recours à la congolité comme argument de vente.

$\mathrm{Au}$ cours du règne de Joseph Kabila (2001-2019) sous le régime duquel un discours souverainiste a émergé de façon exponentielle contre ce que la «Kabilie » (les partisans de l'idéologie kabiliste, selon le langage ordinaire congolais) appelait à l'époque "l'ingérence extérieure dans les affaires politiques congolo-congolaises ", le Ministère de l'Enseignement Primaire, Secondaire et Professionnel (EPSP) a changé de nom pour devenir Ministère de l'Enseignement Primaire, Secondaire, Technique et Initiation à la Nouvelle Citoyenneté (EPST-INC). Il s'agit d'un changement qui est intervenu après un certain constat de crise de patriotisme au sein du peuple, sans doute désenchanté par la praxis du mode de gouvernance d'une élite politique prédatrice d'un peuple meurtri qu'elle gouverne. D'où une campagne de sensibilisation citoyenne au "patriotisme » et au «nationalisme», deux valeurs cardinales que doit incarner tout digne citoyen.

Cette nouvelle donne s'est vite élargie à l'univers discursif de la publicité. Dès lors, le discours publicitaire pour la vente des objets classiques, tels que les boîtes mathématicales, les cahiers, etc. encodent des signes sémiotiques d'énonciation de la congolité, en tant que celle-ci constitue un argument de vente c'est-à-dire un moyen de persuasion. Il s'agit, entre autres aussi, des signes chromatiques de référence aux couleurs $\mathrm{du}$ drapeau national (bleu-jaune-rouge). Par exemple, un type d'objet classique, appelé "Cahier ministre" dans le jargon scolaire, porte une couverture au sceau des couleurs du drapeau national sur le devant ainsi qu'aux portraits des deux héros nationaux, à savoir Patrice Emery Lumumba et Laurent-Désiré Kabila, au verso : 


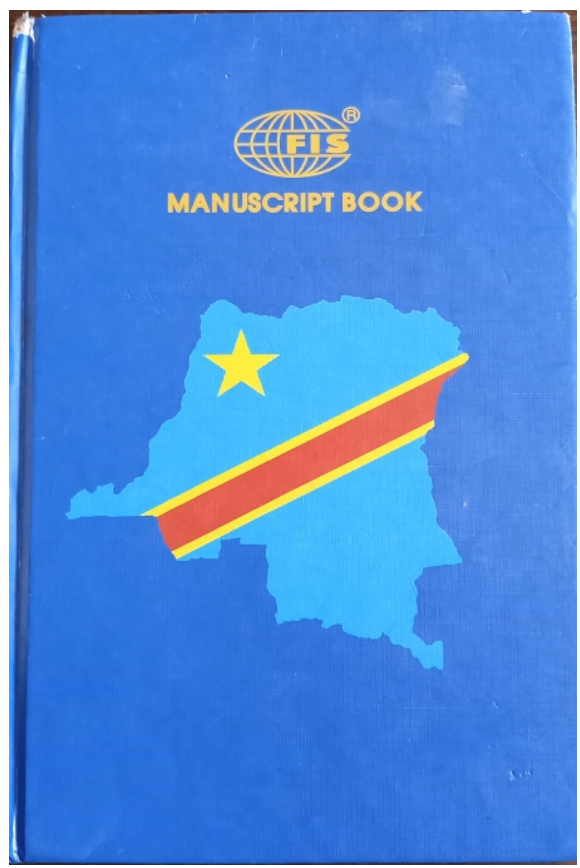

Figure 6. Couverture de cahier Ministre (recto)

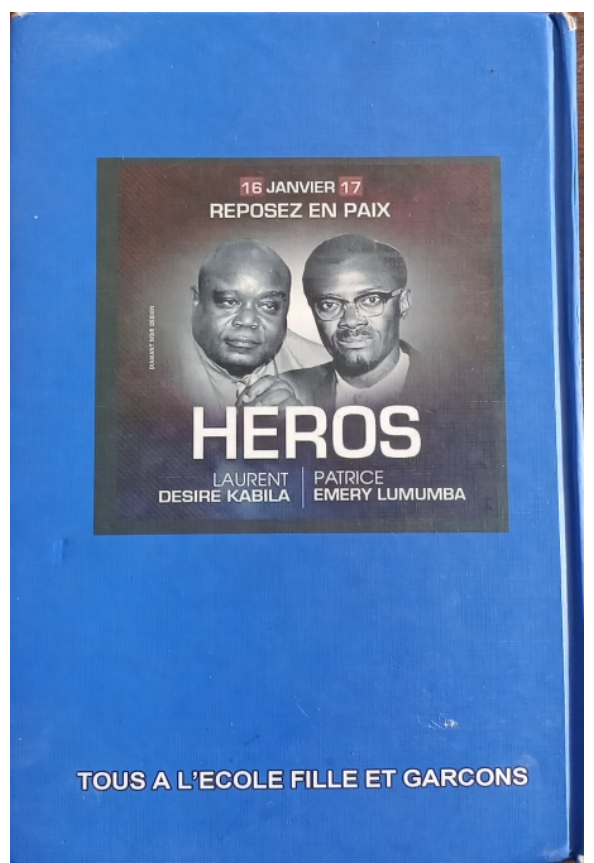

Figure 7. Couverture de cahier Ministre (verso)

Ces deux modèles d'héroïsme nationaliste et de patriotisme ont été respectivement assassinés le 17 janvier 1961 et le 16 janvier 2001. Ils constituent deux principales figures emblématiques de résistance nationaliste contre l'impérialisme colonial et l'invasion du territoire national par des armées étrangères, mais aussi contre l'oppression de la dictature de Mobutu Seseseko. La presque coïncidence des dates de commémoration de leur mort chaque année, mais aussi leur quasisimilarité en discours, en action et en idéaux ont permis le rapprochement idéologique de leur parcours politique et de leur destin dans l'histoire générale de la RD Congo.

Dans d'autres cas de figure, comme par exemple celui du «Cahier de dessin », l'élan d'exaltation patriotique de la «Nation», à travers la " congolité » mise en discours en tant que valeur marchande, est exprimé par des iconotextes aux portraits du Président de la République, des Léopards c'est-à-dire les joueurs de l'équipe nationale de football, au lendemain de leur historique victoire finale au Championnat des Nations (CHAN/Rwanda), organisé au Rwanda en 2016. Et le tout se trouve sur un fond de la couverture du cahier arborée tout aussi aux couleurs du drapeau national : 


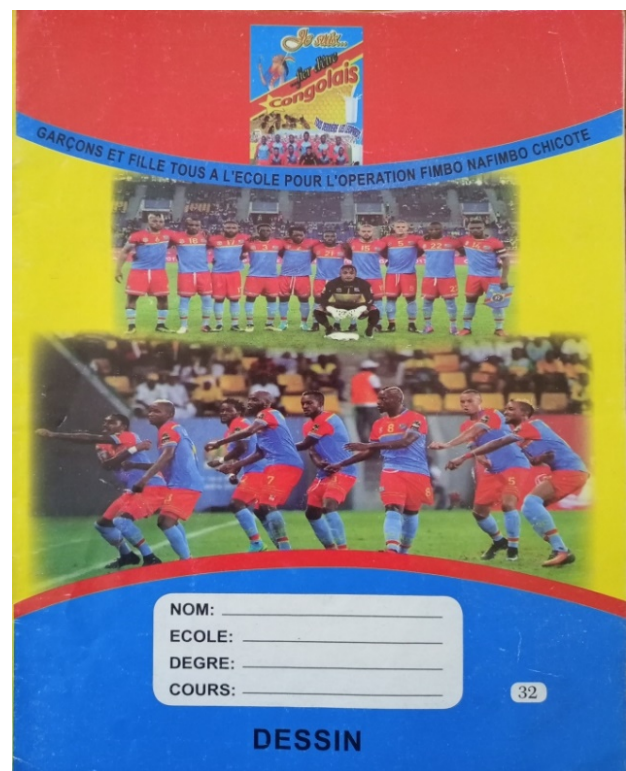

Figure 8. Cahier de dessin

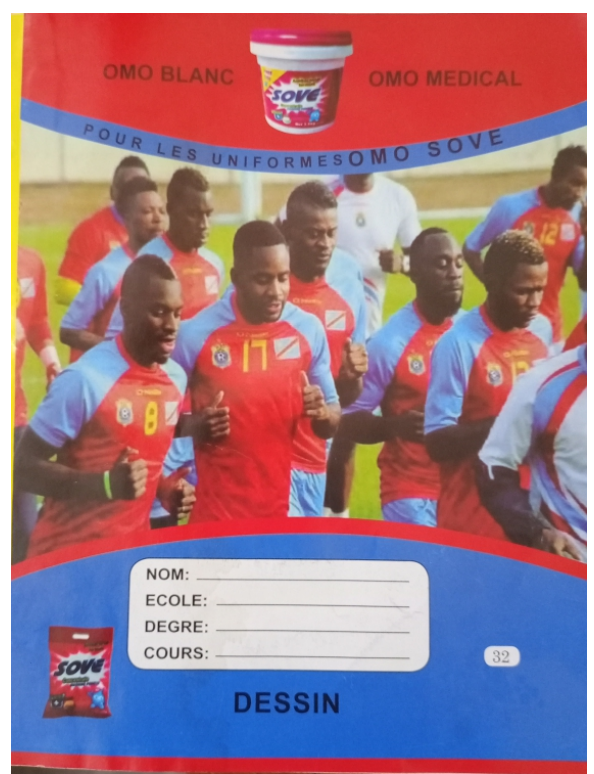

Figure 9. Cahier de dessin

La Figure 8 représente les Léopards en mode de célébration, au rythme de leur mythique danse «Fimbu na Fimbu, Chicotte». Elle comporte également deux énoncés verbaux à savoir le slogan de publicitaire de la congolité, "Je suis fier d'être Congolais » ainsi qu'un autre slogan féministe «Garçons et filles, tous à l'école pour l'opération Fimbu na Fimbu chicotte » qui ne nous intéresse pas dans le cadre de cette étude. Par contre la Figure 9, qui met en scène les mêmes joueurs de l'équipe nationale comporte aussi une boîte et un message publicitaire pour le savon "Omo sove », une qualité d'omo blanc et médical. C'est le message : «Pour les uniformes OMO SOVE».

En effet, les Léopards avaient remporté cette coupe du CHAN au terme d'une finale disputée contre le Mali, battu par un score sans appel de trois buts à zéro. En plus, ce trophée avait été gagné après avoir éliminé le Rwanda, pays organisateur de la compétition, en quart de finale. Cette élimination du Rwanda par les Léopards avait été aussi saluée comme une victoire politiquement symbolique contre le "Voisin mabe" (mauvais voisin, en lingala), un expression devenue idiomatique pour désigner le Rwanda dans le langage courant des Congolais. D'ailleurs, la veille de ce match contre le Rwanda, le sélectionneur national des Léopards avait déclaré dans une interview sur la voix des ondes: «Nous allons jouer pour nous-mêmes et pour ceux qui sont morts à l'Est». C'est une déclaration 
éminemment politique car elle fait écho à une certaine opinion partagée, selon laquelle le Rwanda est à l'origine de plus de cinq millions de morts enregistrées à l'Est du pays, sous-couvert de fausses rébellions à tendance ethnique rwandophone. C'est dans cette optique qu'il y a lieu de parler de la géopolitique du sport comme arme de guerre diplomatique.

Par ailleurs, ces performances sportives des Léopards A au CHAN/Rwanda 2016 sont intervenues juste une année après celles obtenues par les Léopards A qui avaient remporté le trophée de la troisième place à la Coupe d'Afrique des Nations (CAN)/Guinée Équatoriale 2015. Elles ont été célébrées comme un élément fédérateur des tous les Congolais autour de la valeur-Nation, au-delà des clivages politiques à coloration tribalo-ethnique et régionaliste. C'est notamment l'exemple du clivage Est-Ouest, de plus en plus manifeste depuis l'élection de 2006, au cours de laquelle le Président Joseph Kabila Kabange, candidat de l'Est swahiliphone avait remporté le scrutin au deuxième tour devant l'ancien Sénateur Jean-Pierre Bemba Gombo, candidat de l'Ouest lingalaphone. Ainsi la mobilisation des Congolais autour de leur équipe nationale a dès lors été instrumentalisée par la classe politique au pouvoir comme le symbole de l'unité nationale et de cohésion sociale pour rassembler le peuple de plus en plus hostile à un régime prédateur, et très pessimiste quant au devenir de leur pays en proie à des crises multiformes.

Toujours dans le même contexte, certains produits (objets scolaires, calendrier, T-Shirts, etc.) au portrait du feu Colonel Mamadou Ndala Moustapha, assassiné la 2 janvier 2014 lors d'une embuscade à Beni, la zone des opérations militaires «Sokola I » (Nettoyer), abondent de plus en plus sur le marché. Aujourd'hui, ce «vaillant» Colonel des FARDC (Forces Armées de la RD Congo) a été élevé au rang de «héros national » au sein de l'opinion commune pour la brillante victoire de son bataillon/unité contre la rébellion du M23, appuyée par les armées rwandaises et ougandaises à l'Est du pays. Cette victoire est désormais restée gravée dans la mémoire de tous les Congolais comme un symbole de vengeance de la RD Congo contre le voisin-ennemi, le Rwanda dont l'armée avait fait subir des défaites cinglantes à son armée pendant les guerres de rébellions menées jadis par le RCD/Goma (1998) et le CNDP (2008).

En leur temps, ces deux rébellions, essentiellement mono-ethniques rwandophones, avaient été accusées d'être au service d'un projet machiavélique d'extension de l'«Empire Hima tutsi » (Tutsi Land) sur le territoire congolais. À ce point, le Colonel Mamadou Ndala incarne le 
modèle de commandement d'une armée congolaise puissante. Observons à cet effet les images ci-dessous :

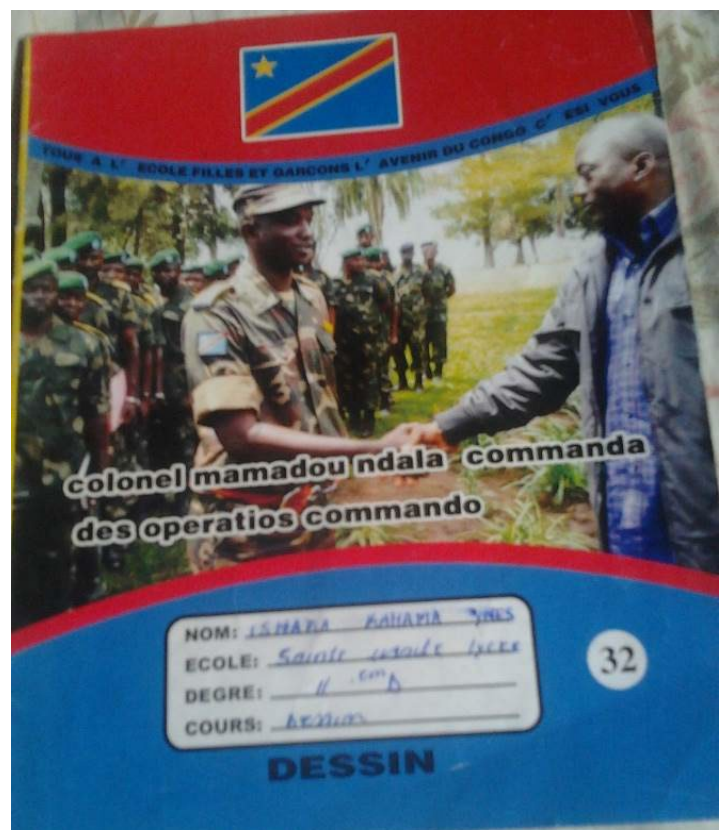

Figure 10. Colonel Mamadou sur Cahier

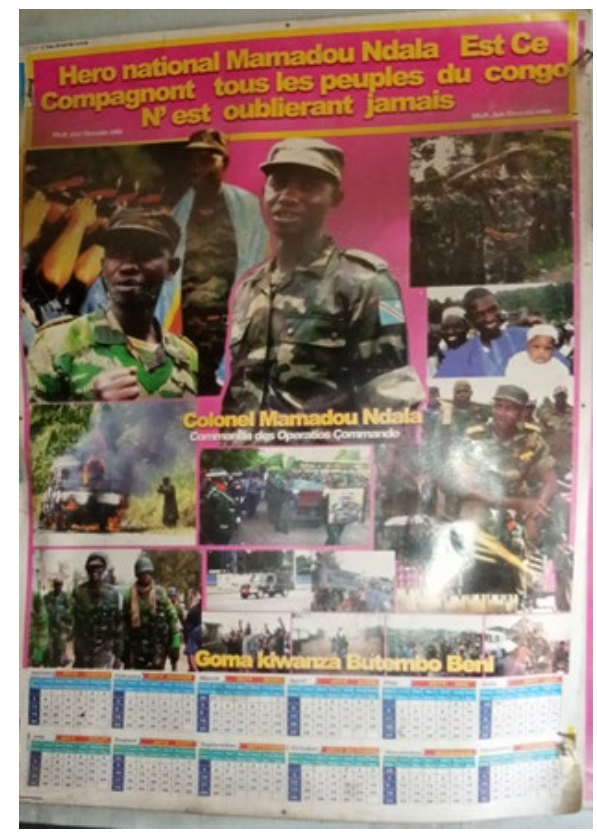

Figure 11. Colonel Mamadou sur calendrier

La figure 10 ci-dessus, prise sur un cahier de dessin, nous montre le Colonel Mamadou Ndala Moustapha accueillant l'ancien Président Joseph Kabila Kabange qui fait le tour des troupes de son unité dans la zone des opérations commando à BENI (Nord-Kivu). Par contre, la figure 11 le représente en différents contextes de son parcours professionnel sur un calendrier, avec un message fort significatif, quoi que truffé de fautes de langue : "Héro national Mamadou Ndala et ses compagnons. Tous les peuples du Congo ne t'oublieront jamais! ». L'enjeu d'un tel discours est sans doute de pérenniser les noms de ces figures politiques dans la mémoire collective nationale car elles constituent des modèles d'héroïsme pour tout citoyen, notamment la jeunesse. Le portrait de Mamadou Ndala fait aussi parfois la une sur la toile des réseaux sociaux, tel que nous l'illustre le cas présent de l'internaute Nyamazi Fredy Mihigo. La photo est accompagnée d'un message verbal qui en explicite le sens. Il s'agit du message « Le symbole $d u$ courage et de la détermination! MAMADOU NDALA Moustapha»: 


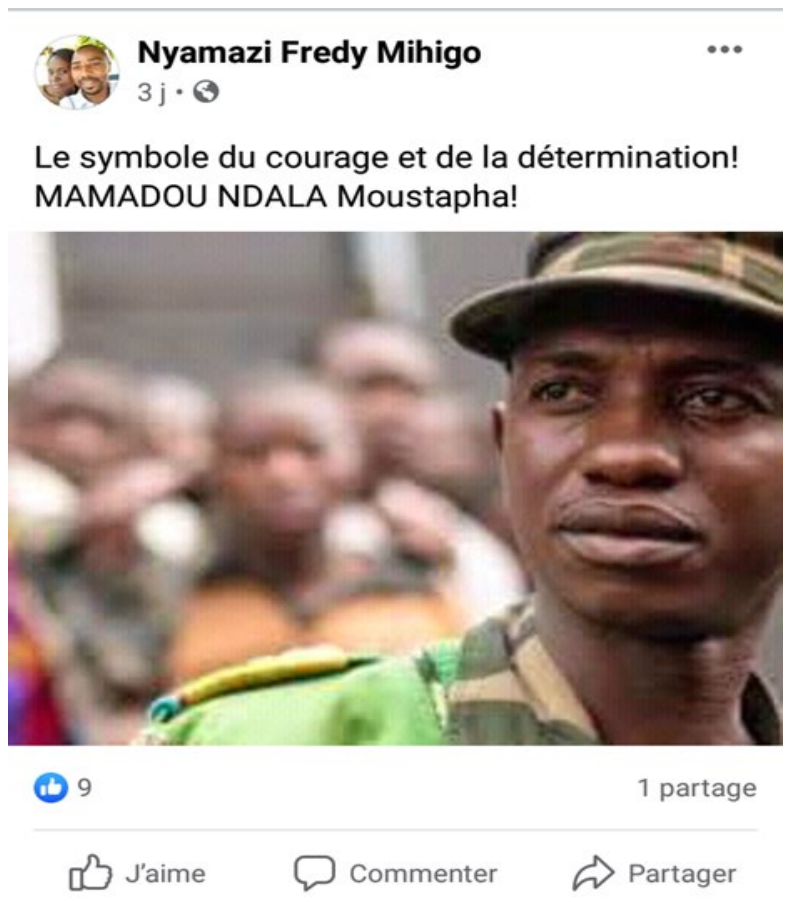

Le personnage du Colonel Mamadou Ndala est de plus en plus évoqué au sein de l'opinion publique comme le modèle de bravoure et de patriotisme. Son nom est parfois aussi évoqué au sens positif de personnalité de probité morale et de responsabilité citoyenne pour avoir refusé, à maintes reprises, des sommes colossales d'argent afin de ne jamais trahir la patrie. Cette boîte mathématicale ci-dessous est tout aussi marquée de ces codes sémiotiques de nature chromatique. Elle donne à voir le drapeau du pays dans le fond duquel il est écrit aux lettres des mêmes couleurs de ce drapeau l'énoncé «Je suis ... fier d'être Congolais » :

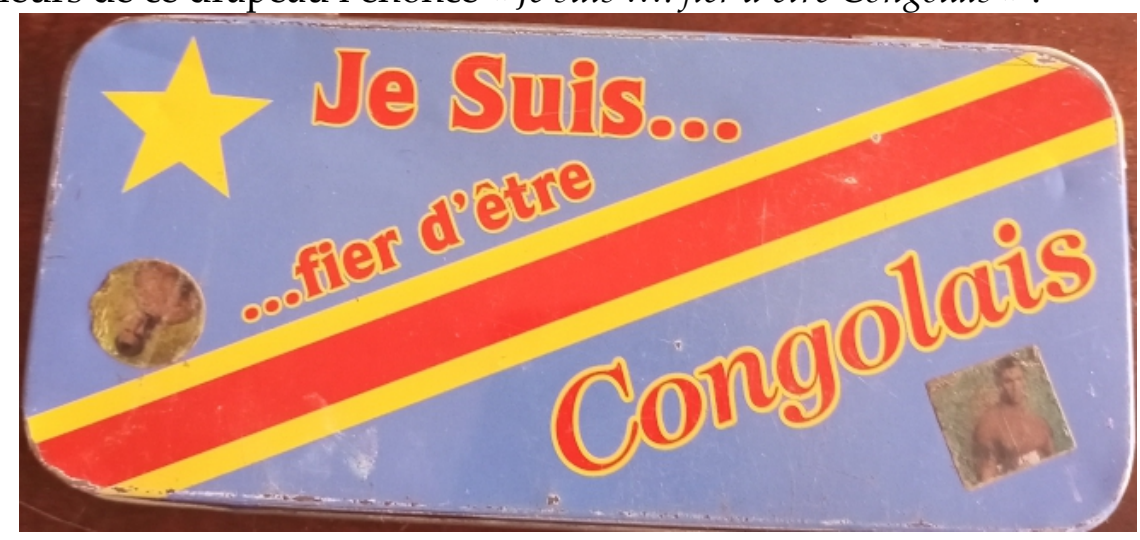

Figure 12. Boîte mathématicale 
Dans la même dynamique, les performances sportives de l'équipe nationale à la CAN/Guinée Équatoriale 2015 et au CHAN/Rwanda 2016 ont aiguillonné le sentiment de fierté et d'appartenance à une "grande Nation", au point qu'elles ont donné lieu au marconyme "Bonbon Léopards ", si nous devons éviter le terme générique d'ergonyme. Tous ces codes sémiotiques (images/couleurs du drapeau national) sont parfois accompagnés de l'énoncé verbale «Je suis fier d'être Congolais » qui leur sert de « cotexte » (Maingueneau, 2009: 41-42), en étayant le sens implicite et la «visée argumentative" (Amossy, 2010) c'est-à-dire qui en oriente l'interprétation. Dans le champ de l'école tout comme ailleurs, ces produits commerciaux portant le sceau de la «congolité » bénéficie d'une large audience au sein de la clientèle nationale.

Dès lors, la congolité se trouve figurativisée par une relation référentielle indicielle qui permet de déduire la façon dont son discours $s^{\prime}$ articule à son contexte de production et de signification. C'est autant dire que la convocation implicite ou explicite de la "congolité », comme « ontotype» de patriote nationaliste, entraîne des effets manifestement modalisants, relevant de la représentation sur lui-même et sa clientèle. Sous un autre angle, nous pouvons déduire avec Charaudeau, P. (2001: 341-348) le fait que la congolité mobilise, entre autres, "les savoirs de croyances qui correspondent aux systèmes de valeurs plus ou moins normés, qui circulent dans un groupe social [...], alimentant les jugements de ses membres et qui et qui en même temps, donnent en même temps, donnent à celui-ci sa raison d'être identitaire ». Et ces savoirs de croyances n'ont pas comme supports les seuls signes iconiques. Ils s'énoncent également à travers le pouvoir des mots relevant du code verbal.

\subsection{L'expression verbale de la congolité}

À côté des signes iconiques, la «congolité » est aussi mise en discours au moyen des signes verbaux. Ceux-ci concernent quelques énoncés (slogans) qui comportent des mots du champ lexical du paradigme congolais. La stratégie discursive mise en œuvre consiste à présenter le « consommer/acheter congolais » comme la condition sine qua non d'être Congolais. Certains de ces énoncés du corpus présentent l'acte "Achetercongolais » comme une preuve de patriotisme sans lequel le client se voit symboliquement perdre sa congolité. 
En voici quelques exemples :

(+) Avec Primus, retrouvez la congolité (Chanson Primus de l'artiste Werrason).

(+) Congo mon pays, Airtel mon réseau (Société Airtel).

(+) Congo na motema, Primus na maboko [Congo dans le cœur, Primus dans les mains]

(Publicité pour la bière Primus de la Bralima).

(+) To lova RDC, to pona Primus [Nous avons aimé la RDC, nous avons choisi Primus]

(Publicité de la bière Primus).

(+) Vin Ushindi, force ya bana mboka [Vin Ushindi, la force des patriotes authentiques Ushindi).

(Publicité du journaliste Hubert Furuguta pour le vin local

(+) Lait Cowbell, fiers d'être Congolais! (Publicité pour le lait Cowbell).

(+) J'aime la RDC, j'utilise UB-Box (slogan publicitaire pour le décodeur UB-Box de

la Maison Ushindi Business/Goma).

Ce dernier énoncé est celui qui est le plus souvent marqué comme enseigne sur les bâtiments de différents points de recharge de l'abonnement aux chaînes numériques de ce décodeur, tel en témoigne l'image ci-après :

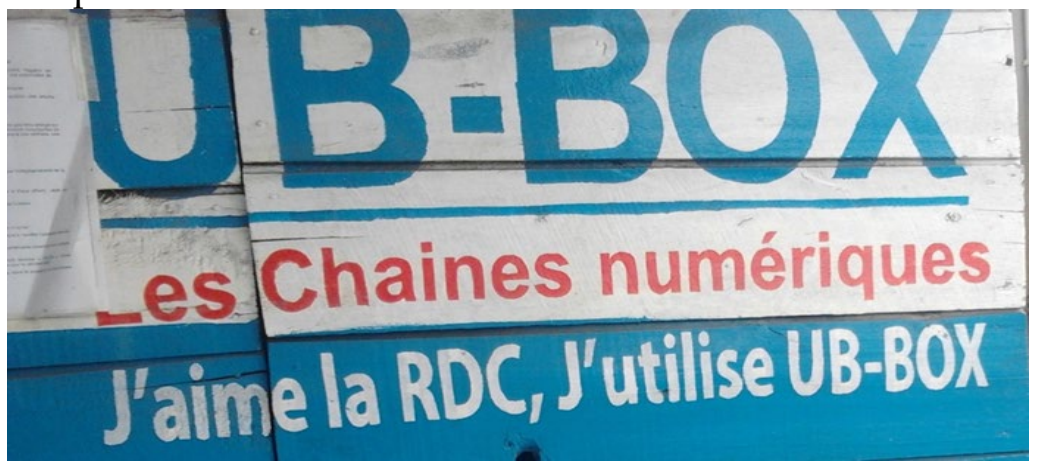

Figure 13. Affiche publicitaire du décodeur UB-Box

Une autre partie des énoncés place plutôt un focus sur la qualité supposée du produit «made in DR Congo » comme argument de vente. Il $\mathrm{s}^{\prime}$ agit des cas ci-après :

(+) Primus, la bière ya ba Congolais! [Primus, la bière des Congolais] (Publicité pour 
la bière Primus de la Bralima).

(+) Avec les produits Ushindi, consommons congolais! (Publicité pour les produits des Établissements Ushindi/Goma).

(+) CCC, le réseau congolais! (Publicité pour la société de téléphonie mobile Congo

Chine Télécom/CCT, rachetée par l'opérateur français Orange).

$(+)$ Les savons SICOVIR, savons de confiance du peuple congolais (Publicité pour le savons de la Société SICOVIR).

(+) Savon SCOVIR, produit congolais, par les Congolais et pour les Congolais!

(Publicité pour les savons de la Maison SICOVIR).

(+) UB-Box, le décodeur $100 \%$ congolais! (Publicité pour le décodeur UB-Box de la

Maison Ushindi Business).

(+) UB-Box, le décodeur congolais! (Publicité pour le décodeur UB-Box de la Maison

Ushindi Business).

(+) Vin Rafiki, le vin de tous les Congolais! (Publicité pour le Vin Rafiki sur la radio

UB FM).

(+) Vin Maisha, un produit $100 \%$ congolais! (Publicité pour le vin Maisha sur le radio UB FM).

Dans ces intertextes implicites et explicites, la «congolité » est énoncée telle qu'elle se fait écho dans les pratiques urbaines de la sémiosphère commerciale. De toute évidence, la sémiotisation de la congolité constitue un cas indiscutable d'illustration de l'instrumentation de la fibre nationaliste comme enjeu de l'empathie publicitaire. Du coup, le publiciste masque à dessein, sinon il essaie de taire les qualités du produit ou de la marque qu'il propose sur le marché pour ainsi centrer son discours sur un paradigme qui consiste à jouer sur les croyances partagées au sein de l'opinion nationale, sa clientèle cible. Et c'est tout cet ensemble signifiant qui entérine l'idée de la pubosphère comme «espace interactif » entre le vendeur et le client. Or selon le point de vue de Vion (1992: 112), «un espace interactif se construit à tout moment dans et par les activités discursives, les choix lexicaux, les attitudes, les manières de s'impliquer ou d'interpeller ». Du coup, les formes, couleurs, mots, figures politiques représentées dans l'histoire du pays comme des modèles de nationalisme 
constituent des faits c'est-à-dire des signifiants culturels de nature symbolique.

\section{La congolité comme rhétorique de populisme nationaliste}

Le populisme constitue sans doute l'enjeu ultime de $l^{\prime}$ « hyperpublicitarisation» de la «congolité » dans la communication marchande. Son instrumentation dans le discours publicitaire ouvre en cela la voie à une tendance qui fonde ce dernier sur des jugements a priori c'està-dire sur des préjugés en circulation autour du produit soumis sur le marché. Elle en constitue un facteur de catalogage et d'étiquetage. Aussi la congolité constitue-t-elle un cas de «stéréotype (Amossy et HerschbergPierrot, 1997) pour désigner un processus d'appréhension artificielle de soi et d'autrui. Elle confère in fine au produit, des qualités authentiques en vue de l'assurance des esprits de la clientèle (Yücel, 2013). En effet, un discours qui sublime les produits «made in DR Congo » c'est-à-dire des "produitsNation » ne peut qu'ouvrir la voie au populisme. Celui-ci adopte à son tour les formes d'ancrage identitaire pour la fabrique d'un ethos collectif de patriote.

\subsection{La congolité comme ancrage identitaire d'un discours populiste}

Le discours est le lieu par excellence où se construisent et se déconstruisent les identités. La « congolité » relève d'une identité culturelle et politique qui se décline comme la «valeur de base » de toute proportion de vente. Tel que nous l'avons identifié dans les textes, le fait d'être Congolais va de pair avec la fierté. Le relent patriotique y est induit par le discours qui l'accompagne car elle implique une image de nationaliste, supposée partagée par tous. Étant donné que tout discours s'élabore en fonction des caractéristiques d'un public cible donné, la «congolité » comme signe ou système de signes actualisés dans le discours publicitaire congolais ne se construit pas de façon unilatérale par le publiciste-émetteur mais elle suppose la mise en œuvre du principe de co-construction du sens, fondé sur la prise en compte du destinateur dans le processus communicatif.

Du coup, il en résulte la mise en jeu du principe d' « intérêt » et de "communion affective ou collective » (Guiraud, 1971: 18) autour de l'identité nationale. C'est dans cette optique d'ancrage identitaire qu'il faut 
lire les slogans publicitaires "Congo mon pays, Airtel mon réseau » de la maison de téléphonie mobile Airtel, mais aussi «To lova RDC, to pona Primus » (Nous avons aimé la RDC, nous avons choisi Primus » de la bière «Primus» de la société Bralima). Dans ce sens, "Acheter/consommer congolais " devient une confession au nationalisme, un devoir honorable pour tout citoyen congolais. Dans le même ordre d'idées, "Ne pas acheter/consommer congolais » constitue un acte de haute trahison, par lequel l'acheteur inféode la Nation à l'impérialisme économique néocapitaliste.

Dans sa manifestation discursive en communication publicitaire, la congolité suppose la co-construction de trois types d'identités. Il y a l'identité du produit mis en vente pour trois formes de congolité, celle du vendeur et celle du client. La congolité de la marchandise en vente repose sur sa qualité de "produit made in DR Congo", celle du vendeur sur son sentiment et son faire patriotiques de "Vendre congolais » et celle du client sur son «pouvoir-faire» tout aussi patriotique $\mathrm{d}^{\prime}$ «Acheter/consommer congolais ». Ainsi, "Ne pas acheter/consommer congolais» devient-il un acte sentencié par lequel le client se rend automatiquement coupable d'incivisme.

D'un point de vue purement idéologique, le produit trouve sa légitimité sur le marché dans et par cette forme implicite de revendication identitaire. Par ailleurs, grâce à son discours et à son action, le publiciste transforme l'acte "Acheter/consommer congolais » en un puissant bastion de résistance patriotique contre l'impérialisme sino-occidentalo-américain, sur le modèle duquel se régule le circuit du commerce international. En même temps, il s'agit de l'expression d'un sentiment identitaire fort et d'engagement pour la défense d'un patrimoine-nation commun. Et pour reprendre les termes de Dalila Hazi (2013), il y a lieu de dire de cette nouvelle configuration de la pratique publicitaire que :

"Elle est due à l'articulation de dynamiques de conscientisation qui portent sur de nouvelles formes énonciatives et discursives ainsi que sur les formes de gestion, de différentiation et d'ajustement identitaires. »

Dans la mesure où l'instance énonciative (publiciste) a l'intention de toucher aussi bien les cœurs que les esprits de la clientèle cible, son discours relève alors du type de "communication grégaire» (Eloundou Eloundou, 2013), car selon ses termes :

"Étant donné que tout discours publicitaire est susceptible d'agir sur le potentiel consommateur, nous avons des enjeux pragmatiques, sémanticosymboliques et communicationnels qui fonctionnent en synergie pour 
donner une force illocutoire au discours par des contenus implicites ou latents. »

Enfin de compte, le pouvoir manipulateur du discours de la " congolité » consiste à présenter celle-ci comme le seul gage de qualité et d'authenticité, au-delà des valeurs intrinsèques du produit proposé pour la vente. De ce côté, il s'apparente à une sorte de stratégie "pernicieuse » relevant de la propagande. C'est alors un discours d'emprise psychologique à élan accrocheur et vis-à-vis duquel aucun «vrai patriote congolais » ne saurait rester impassible. Ce discours publicitaire s'envisage désormais en termes de discours populiste qui instrumentalise la fibre nationaliste en tant que valeur marchande dans la praxis économique. D'ailleurs, l'énoncé «J'aime la $R D C$, j'utilise $U B$-Box» nous semble avoir été calqué sur le modèle du slogan politique "J'aime la $R D C$, je soutiens Vital Kamerhe». Ce dernier avait en effet été élaboré comme message de campagne de Vital Kamerhe lors de la présidentielle de 2011.

Pour ne prendre que l'exemple des slogans «Avec Primus, retrouvez la congolité », "Congo mon pays, Airtel mon réseau ", "J'aime la RDC, j'utile $U B-B o x$ », la notion de " congolité » encode des contenus socio-sémantiques implicites, dotés d'une charge illocutoire à empathie axiologico-affective dans ses manifestations au sein du corpus. Elle constitue un " hypercode » langagier qui traduit, à dessein, un certain syncrétisme identitaire construit autour de la valeur-nation. C'est dans cette optique d'analyse que la « congolité » comme paradigme culturel, politique et idéologique est à appréhender en termes de représentation du monde, à la fois perçue, construite et orientée par le contenu modal linguistique du discours qui en est le vecteur, selon la perspective de la Sémantique des Possibles Argumentatifs (SPA) que propose à Galatanu, O. $(2003,2009)$. En plus, elle ouvre la voie à une «opinion partagée » (Amossy, 2010 : 97) qui fonde le discours publicitaire sur des jugements a priori c'est-à-dire sur des préjugés qui circulent autour du produit. Toutefois, la visée reste la fabrique d'un ethos collectif de vendeur/consommateur nationaliste.

\subsection{La congolité comme mis en scène de l'ethos collectif de nationaliste}

La co-construction d'un ethos de patriote Congolais est au cœur de tous les enjeux du discours publicitaire de la congolité. Par l'intrusion d'une telle donne dans la sémiosis économique, le discours de la congolité donne à voir aux « consommateurs hédonistes » (Sintas, 2013), des produits 
nationaux authentiques, "made in DR Congo », en tant que "marques-pays » autour desquelles se construit l'identité collective authentique des vrais Congolais. Les exemples les plus remarquables à citer, de par leur prégnance, ce sont entre autres des expressions idiomatiques sur certains produits et devenues figées dans le langage ordinaire de la publicité brassicole.

Autour de la bière «Primus» de la société Bralima, il faut retenir des expressions telles que "La bière ya bana mboka " (La bière des nationaux c'est-à-dire des patriotes nationalistes, en lingala), «La bière du cinquantenaire de l'indépendance», mais aussi et surtout "Avec Primus, retrouvez la congolité » (chanson de l'artiste musicien Ngima Makanda dit Werrason pour la publicité de la bière "Primus»). Dans le secteur de la téléphonie mobile, il faut mentionner le slogan «Congo mon pays, Airtel mon réseau ». Par ailleurs, le seul exemple à retenir dans le secteur de la publicité des institutions bancaires et de microfinances c'est l'énoncé en anglais "Léopards is my team, Raw Bank is my bank» (Léopards est mon équipe, Raw Bank est ma banque). Bref, le discours de la «congolité » est un discours au relent à la fois identitaire et politique qu'il faut inscrire, idéologiquement parlant, dans le projet global de la campagne «Consommons congolais! » lancée depuis 2013.

C'est à partir de la convocation des valeurs de "patriotisme » et de « nationalisme » que se crée un « espace interactif » où les potentiels clients se sentent directement interpellés par l'énonciateur à travers l'objet de sa communication. Du fait que le processus a pour finalité la réalisation d'un «Faire-acheter/consommer congolais », nous envisageons le paradigme de la « congolité » comme source des stratégies publicitaires (Harir et Bakrim, 2013), en tant que forme sémiotique à enjeu $d^{\prime}$ " «axiologisation discursive » (Prieto, 2015). Celle-ci est par ailleurs construite autour des formes modales essentiellement déontiques qu'elle sous-tend dans leur environnement sémantique et pragmatique. Le discours sur la congolité relève de la rhétorique de l'éloge comme forme d'argumentation publicitaire (Adam et Bonhomme, 1997) car il consiste en une persuasion sublimale.

Selon Greimas, A.-J. et Courtés, J. (1979: 90), « du point de vue sémiotique, la structure modale déontique apparaît lorsque l'énoncé modal, ayant pour prédicat le devoir, surdétermine et régit l'énoncé de faire ». Dès lors, la «congolité » est un paradigme-valeur doublement modélisé car au «Devoir-produire/vendre congolais » du marchand correspond un «Devoir- 
acheter/consommer congolais » du client pour ainsi donner lieu à deux formes de carré sémiotique de catégorie modale déontique :

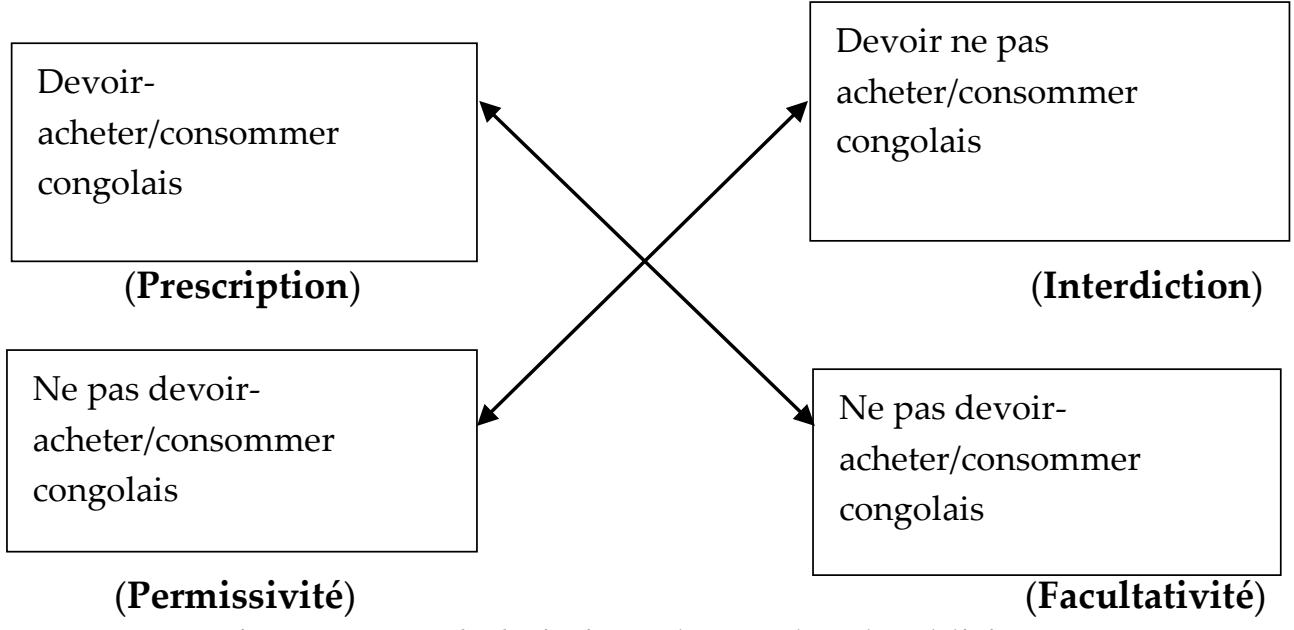

Figure 14. Carré sémiotique du marchand-publiciste

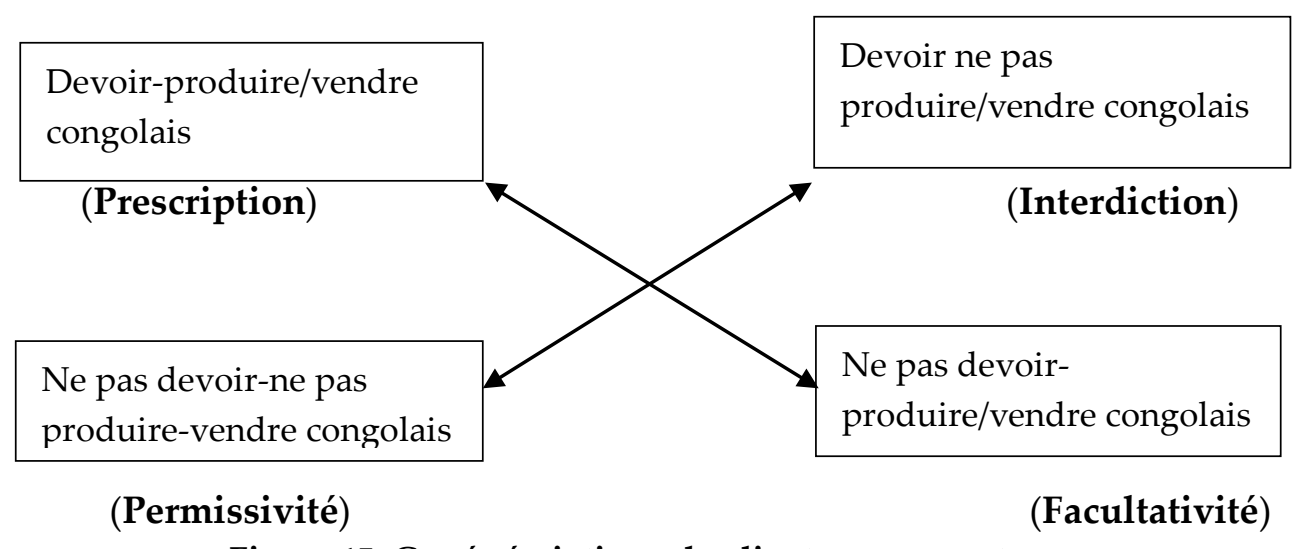

Figure 15. Carré sémiotique du client-consommateur

Dans cette dynamique de rhétorique populiste, évoquer le risque de perdre sa "congolité » c'est-à-dire sa nationalité congolaise symbolique pour le simple fait de ne pouvoir pas réaliser le faire performatif "Acheter/consommer congolais" peut alors s'envisager comme une forme d'argumentation coercitive. Il implique un phénomène $d^{\prime}$ " acte menaçant pour la face négative » (Brown et Levinson, 1978) de l'allocutaire-client, tel que Maingueneau (2009: 62) nous en présente la synthèse. Dans un contexte géopolitique où il faut mobiliser la nation en posture victimaire 
contre une invasion étrangère multiforme, la « congolité » est à prendre au sens d'« idée reçue » (Amossy, 2010 : 97) du fait de sa valeur coercitive et injonctive. L'enjeu coercitif consiste à contraindre le client à réaliser l'acte induit par le discours s'il ne veut pas perdre sa face positive, car le « Devoiracheter/consommer congolais » est ici posé comme une obligation morale c'est-à-dire une contrainte d'ordre éthique. D'où les deux pôles opposés à savoir "Acheter/consommer congolais" comme acte de civisme et «Ne pas acheter/consommer congolais » comme acte d'incivisme marchand.

Selon le schéma de persuasion adopté, l'application de la notion de "syllogisme » peut intervenir ici pour la reconstruction de l' " enthymème » dans la communication argumentative (Amossy, $2010: 112)$ sur la congolité en publicité. Prenons par exemple le cas de "Avec les produits Ushindi, consommons congolais ". Cet énoncé correspond à une mineure formulée, à partir de laquelle le syllogisme peut être reconstitué dans l'optique de la congolité comme qualité de "vrai congolais»: "Consommer congolais c'est être un vrai Congolais (majeure fondée sur un lieu non formulé), Avec les produits Ushindi, consommons congolais (mineure formulée). Donc consommer les produits Ushindi c'est être un vrai Congolais (conclusion non formulée). Le destinataire du message publicitaire doit donc chaque fois passer par ce processus afin de pouvoir inférer le sens et la visée pragmatique du discours qui lui est adressé. C'est l'interaction de tous ces ingrédients qui structure le processus persuasif $\mathrm{du}$ «Faire acheter congolais ».

\section{Conclusion}

Cette étude a consisté à cerner tous les enjeux du paradigme de la « congolité » en tant que forme rhétorique au discours qui relaie, implique et structure les croyances autour des valeurs de nationalisme et de patriotisme en RD Congo. L'analyse nous a permis d'inférer les ressorts pathémiques du discours de propagande nationaliste dans la publicité commerciale, constituée de constructions identitaires aux enjeux commerciaux et politiques. Il a été établi que la "congolité » fonctionne comme un « possible argumentatif » d'exaltation patriotique au prisme de son axiologisation discursive dans le corpus. Aussi constitue-telle une forte empathie d'accroche du destinataire cible qu'est le Congolais patriote car «acheter/consommer congolais» devient désormais un gage de digne Citoyen idéal c'est-à-dire de citoyenneté responsable pour l'essor de l'économie nationale. Par son discours, l'énonciateur du slogan et/ou de 
l'affiche publicitaire entend instituer la "congolité » en une "valeur doxologique » et en une "règle déontique » (Prieto, 2013) c'est-à-dire en un « ontotype » de patriotisme commercial en RD Congo.

\section{Références bibliographiques}

1. ADAM, J.-M. et BONHOMME, M. (19970, L'argumentation publicitaire. Rhétorique de l'éloge et de la persuasion, Paris : Nathan.

2. AMOSSY, R. (2010), L'argumentation dans le discours, 3e édition. Paris : Armand Colin.

3. AMOSSY, R. et HERSCHBERG-PIERROT, A. (1997), Stéréotypes et clichés : langue, discours, société, Paris : Nathan.

4. CHARAUDEAU, P. (2021), "Langue, discours et identité culturelle». CAIREN, $\mathrm{N}^{\circ} 123$, revue électronique disponible [en ligne], disponible sur le site: https://www.cairen.info/article.php?, consulté le 22 avril 2021.

5. ELOUNDOU ELOUNDOU, V. (2021), «Éléments culturels dans le discours publicitaire au Cameroun: Jeux et enjeux». Les identités culturelles dans le discours publicitaire. Signes, Discours, Sociétés, $\mathrm{n}^{\circ} 11$, 17 juillet 2013. Les identités culturelles dans le discours publicitaire, [en ligne], disponible sur le lien: http://www.revue-signes.info/ document.php?id=3116. ISSN 1308-8373, consulté le 20 avril 2021.

6. GALATANU, O. (2003), « La sémantique des possibles argumentatifs et ses enjeux pour le discours ». In : Inãrrea Las Heras, M.-J. Salinero Cascante (éds.). Actes du XXème Colloque de la APFFUE: Congré International de Estudios Franceses, Logronõ (La Rioja), 7-10., La rioja, 2002, Vol. 2 Encrucijada de Caminos, Logrono (La Rioja), 7-10 mai 2002.

7. GALATANU, O. (2007), «Sémantique des Possibles Argumentatifs et axiologisation discursive ». In : Bouchard, D. et I. Evrard, Vocaj, E. (éds). Actes du Colloque international de Montréal : Représentation du sens linguistique II, Université du Québec, Montréal, 23-25 mai 2003. Bruxelles, Deboeck/Duculot, pp. 313-325.

8. MAINGUENEAU, D. (2009), Les termes clés de l'analyse du discours, Nouvelle édition revue et augmentée. Paris : Éditions du Seuil.

9. MAINGUENEAU, D. (2014), Analyser les textes de communication, Nouvelle édition revue et augmentée.

10. MOHAMMEDI, A. (2017), " Rhétorique identitaire et souveraineté au XXe siècle». Le vent se lève, avril 2017 [en ligne] sur : 
htts://lvsl.fr/reflexion-origines-rhetorique-identitaire, consulté le 22 mars 2021.

11. PRIETO, J.-P. (2013), «Axiologisation discursive de représentations identitaires : le cas de la chanson engagée "Dans la jungle " de Renaud Séchan ». Les identités culturelles dans le discours publicitaire. Signes, Discours, Sociétés, n¹5, 15 janvier 2015, [en ligne], disponible sur le lien :

12. SINTAS, S. (2013), «De la promotion des produits régionaux à celle de l'identité nationale catalane ». Les identités culturelles dans les discours publicitaire. Revue Signes, Discours, Sociétés, n¹1, 17 juillet 2013 [en ligne], disponible sur le lien : http://www.revuesignes.info/document.php?id=3114.ISSN1308-8378, consulté le 15 avril 2021.

13. YÜCEL, H. (2013), «L'identité turque dans le discours publicitaire turc ». Les identités culturelles dans le discours publicitaire. Signes, Discours et Sociétés, n¹1, 14 juillet 2013 [en ligne], disponible sur le lien : http://www.revue-signes.info/document.php?id=2956. ISSN 13088373, consulté le 15 avril 2021. 